page-wide by default

\title{
D-branes from Matrix Factorizations *
}

\author{
Kentaro Hori ${ }^{\mathrm{a}}$ Johannes Walcher ${ }^{\mathrm{b}}$

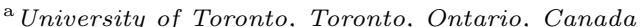 \\ ${ }^{\mathrm{b}}$ Kavli Institute for Theoretical Physics, University of California, Santa Barbara, California, USA ${ }^{1}$
}

\begin{abstract}
B-type D-branes can be obtained from matrix factorizations of the Landau-Ginzburg superpotential. We here review this promising approach to learning about the spacetime superpotential of Calabi-Yau compactifications. We discuss the grading of the D-branes, and present applications in two examples: the two-dimensional torus, and the quintic.
\end{abstract}

\section{Résumé}

Les D-branes de type B peuvent être décrites à partir de factorisations en matrices du super-potentiel de LandauGinzburg. On revoit ici cette approche prometteuse d'apprendre sur le super-potentiel en espace-temps de compactifications de Calabi-Yau. On discute la graduation des D-branes, et présente deux exemples : le tore en deux dimensions, ainsi que la quintique.

Key words: D-branes; Matrix Factorizations ; Superpotential

Mots-clés : D-branes; Factorisations en matrices; Superpotentiel

\section{Introduction}

D-branes wrapped on supersymmetric cycles in Calabi-Yau manifolds have many applications throughout string theory. They have been studied intensively over the last few years from many different points of view, and these studies have led to many remarkable results. One aspect of the problem that is still less understood, at least in practice, is the (effective $4 \mathrm{~d}$ spacetime) superpotential, $\mathcal{W}$, on the worldvolume of such branes. $\mathcal{W}$ being an important quantity for any application, it is worthwhile to look for new ways of computing it. (There are other motivations for the kind of investigation I am undertaking here, but this one should suffice for the moment.)

\footnotetext{
^ Talk given by J.W. at Strings '04, June 28-July 2,Paris

Email addresses: hori@physics.utoronto.ca (Kentaro Hori), walcher@kitp.ucsb.edu (Johannes Walcher)

1 after September 1st 2004 : Institute for Advanced Study, Princeton, New Jersey, USA 
In my talk, I want to describe a new approach to studying D-branes in a certain class of well-known backgrounds, the so-called Landau-Ginzburg models $[1,2,3,4]$. Briefly put, this approach amounts to studying the equation

$$
Q^{2}=W \cdot \mathrm{id},
$$

where $W$ is a polynomial, the Landau-Ginzburg superpotential, which characterizes the closed string background, and $Q$ is an (odd) matrix with polynomial entries, describing the open string configuration. A solution of this equation is called a matrix factorization of $W$.

This approach, the details of which will be discussed momentarily, was proposed in unpublished form by Maxim Kontsevich, and shown to correctly describe the relevant physics, in [5,6,7]. Originally, matrix factorizations go back to [8], and they have since then been studied continuously in the context of singularity theory, which is in fact the mathematical theory underlying Landau-Ginzburg models.

My talk, which is based on [9], and some work in progress, will focus on certain applications of the formalism in the context of $\mathcal{N}=1, d=4$ string compactifications. In particular, I want to show how matrix factorizations of $W$ can lead to (old and new) insights about the spacetime superpotential $\mathcal{W}$, and more generally, about the local and global structure of moduli spaces of D-branes in Calabi-Yau compactifications. Other recent work on the subject includes $[10,11,12,13,14,15,16]$.

\section{Matrix Factorizations}

\subsection{The Warner problem}

To go back to the origin of the problem, I want to consider an $\mathcal{N}=(2,2)$ supersymmetric field theory in two dimensions, of the type that is the starting point for most of perturbative string theory. Let me consider in particular the worldsheet superpotential $W$. To preserve supersymmetry, $W$ is a holomorphic function of the chiral field variables $\Phi$, which (assuming a flat target space) are complex functions on $\mathcal{N}=(2,2)$ superspace with coordinates

$$
x^{+}=t+x, x^{-}=t-x, \quad \theta^{+}, \theta^{-}, \bar{\theta}^{+}, \bar{\theta}^{-}
$$

$\Phi$ satisfies

$$
\bar{D}_{ \pm} \Phi=0
$$

where

$$
D_{ \pm}=\frac{\partial}{\partial \theta^{ \pm}}-i \bar{\theta}^{ \pm} \partial_{ \pm} \quad \bar{D}_{ \pm}=-\frac{\partial}{\partial \bar{\theta}^{ \pm}}+i \theta^{ \pm} \partial_{ \pm}
$$

is the usual covariant derivative. The four supersymmetries are generated by $\mathcal{Q}_{ \pm}, \overline{\mathcal{Q}}_{ \pm}$, where

$$
\mathcal{Q}_{ \pm}=\frac{\partial}{\partial \theta^{ \pm}}+i \bar{\theta}^{ \pm} \partial_{ \pm} \quad \overline{\mathcal{Q}}_{ \pm}=-\frac{\partial}{\partial \bar{\theta}^{ \pm}}-i \theta^{ \pm} \partial_{ \pm}
$$

I want to study this field theory on a space with boundary, say a half-space with boundary at $x^{+}=x^{-}=t$. Translational invariance in $x$-direction being broken, supersymmetry has to be broken also. In geometric terms, this means that superspace acquires a superboundary, which is one-dimensional $\mathcal{N}=2$ superspace, with coordinates

$$
t, \theta, \bar{\theta},
$$

and identified as superboundary via the equations

$$
x^{+}=x^{-}=t, \quad \theta^{+}=\theta^{-}=\theta \quad \bar{\theta}^{+}=\bar{\theta}^{-}=\bar{\theta},
$$

What I have written down here is known as a B-type superboundary, and is invariant under the B-type supersymmetries

$$
\mathcal{Q}=\mathcal{Q}_{+}+\mathcal{Q}_{-}=\frac{\partial}{\partial \theta}+i \bar{\theta} \partial_{t} \quad \overline{\mathcal{Q}}=\overline{\mathcal{Q}}_{+}+\overline{\mathcal{Q}}_{-}=-\frac{\partial}{\partial \bar{\theta}}-i \theta \partial_{t}
$$

(The other possible superboundary consistent with supersymmetry and translational invariance, of A-type, leads to a different problem with a different solution, and I will not consider it here.)

Now an ordinary local field theory, which is invariant under some global bosonic spacetime symmetries, will also be invariant in the presence of a boundary under all symmetries that leave the boundary invariant. This is no longer true for supersymmetries. In the case at hand, the F-term

$$
\int_{\Sigma} d^{2} x d \theta^{+} d \theta^{-} W+\text { c.c }
$$


exihibits a boundary term under the B-type supersymmetry, which is the supersymmetry preserved by the boundary

$$
\begin{aligned}
\int_{\Sigma} d^{2} x d \theta^{+} d \theta^{-} \bar{\epsilon}\left(\overline{\mathcal{Q}}_{+}+\overline{\mathcal{Q}}_{-}\right) W=(\text { using } & \left.\bar{D}_{ \pm} W=0\right) \\
& =\int d^{2} x d \theta^{+} d \theta^{-}\left(-2 i \bar{\epsilon} \theta^{+} \partial_{+}-2 i \bar{\epsilon} \theta^{-} \partial_{-}\right) W=\int_{\partial \Sigma} d t d \theta(-2 i \bar{\epsilon} W)
\end{aligned}
$$

The problem associated with these boundary terms under supersymmetry variations of superpotentials is known as the Warner problem [17].

\subsection{Solution of the Warner problem}

There are various ways of dealing with the Warner problem. (see, e.g., $[17,18,19,20,21,5,6]$ ). One possibility is to introduce boundary conditions that make the boundary term vanish. This has some potentially unwanted consequences such as spontaneously broken worldsheet supersymmetry. Another possibility, which is the topic of present interest, is to work with free boundary conditions, and to introduce additional degrees of freedom living on the boundary, whose supersymmetry variation will cancel the boundary term of the bulk variation. The simplest possibility is to add a boundary F-term

$$
\int_{\partial \Sigma} d t d \theta \Gamma(t, \theta) f(\Phi)
$$

where $f(\Phi)$ is some function of bulk fields (which is chiral on the boundary), and $\Gamma=\Gamma(t, \theta, \bar{\theta})$ is a fermionic superfield on the boundary which fails to be chiral

$$
\bar{D} \Gamma=\left.g(\Phi)\right|_{\partial \Sigma}
$$

where $g(\Phi)$ is some other holomorphic function of bulk fields. It is easy to see that the Warner term is cancelled if and only if

$$
f(\Phi) g(\Phi)=2 i W(\Phi)
$$

This equation, which says that $W$ can be factorized into $f$ and $g$, is the condition that the boundary superpotential preserve $\mathcal{N}=2$ B-type supersymmetry.

\subsection{Consequences}

Under quantization, the Hilbert space of the boundary fermions is simply a two-dimensional vector space $\mathbb{C}^{2}$ graded by Fermion number. In string theory, one will interpret this space as the CP space of a D $\bar{D}$-system, and the chiral fields $f(\Phi), g(\Phi)$ as a tachyon configuration between the brane and the antibrane. More traditionally, one can view $f$ and $g$ as some relevant perturbation of a free boundary condition, much as the bulk superpotential term, $W$. Of course, for string theory, one will have to insure that the induced boundary RG flow reaches a non-trivial IR fixed point, about which I will have more to say a little later.

To find out about the spectrum of open strings, we consider the system on the strip $x \in[0, \pi]$, with solutions of the factorization condition $\left(f_{0}, g_{0}\right)$ and $\left(f_{\pi}, g_{\pi}\right)$ as boundary interactions at the two ends of the strip. As you might vividly imagine, the supercharge will receive a contribution from boundary terms, which acts by a graded commutator

$$
i \bar{Q}_{\mathrm{bdy}}\left(\begin{array}{ll}
a & b \\
c & d
\end{array}\right)=\left(\begin{array}{cc}
0 & f_{\pi} \\
g_{\pi} & 0
\end{array}\right)\left(\begin{array}{ll}
a & b \\
c & d
\end{array}\right)-\left(\begin{array}{cc}
a & -b \\
-c & d
\end{array}\right)\left(\begin{array}{cc}
0 & f_{0} \\
g_{0} & 0
\end{array}\right)
$$

on an open string state with CP structure described by $a, b, c, d$. In particular, the spectrum of supersymmetric ground states is found by studying the cohomology of the operator

$$
\bar{\partial}+i \bar{Q}_{\mathrm{bdy}}
$$

acting on $2 \times 2$ matrix-valued differential forms, which for flat target space amounts to studying $2 \times 2$ matrices with holomorphic entries in the cohomology of $\bar{Q}_{\mathrm{bdy}}$, acting as above. 


\subsection{Example}

The simplest example of all this are the minimal models, with just one variable $\Phi=x$, and superpotential

$$
W=x^{h}
$$

This superpotential can be factorized as $[6,10]$

$$
x^{h}=x^{n} \cdot x^{h-n} \quad \text { for } n=0,1, \ldots h
$$

leading to

$$
Q_{n}=\left(\begin{array}{cc}
0 & x^{n} \\
x^{h-n} & 0
\end{array}\right)
$$

We will denote the corresponding boundary condition by $M_{n}$. The spectrum of chiral operators between $M_{n_{1}}$ and $M_{n_{2}}$ consists of even

$$
\phi_{n_{1}, n_{2}, j}^{0}(x)=\left(\begin{array}{cc}
x^{j-\frac{n_{1}-n_{2}}{2}} & 0 \\
0 & x^{j+\frac{n_{1}-n_{2}}{2}}
\end{array}\right),
$$

and odd operators

$$
\phi_{n_{1}, n_{2}, j}^{1}(x)=\left(\begin{array}{cc}
0 & x^{\frac{n_{1}+n_{2}}{2}-j-1} \\
-x^{h-\frac{n_{1}+n_{2}}{2}-j-1} & 0
\end{array}\right)
$$

where

$$
j=\frac{\left|n_{1}-n_{2}\right|}{2}, \frac{\left|n_{1}-n_{2}\right|}{2}+1, \ldots, \min \left\{\frac{n_{1}+n_{2}}{2}-1, h+\frac{n_{1}+n_{2}}{2}-1\right\} .
$$

As you can see, the branes $n$ and $h-n$ are each others' antibrane, and the branes $n=0, h$ are trivial, as there are no open strings between them and any other brane. This spectrum agrees with the results derived in the rational conformal field theory description of B-type D-branes in $\mathcal{N}=2$ minimal models.

\subsection{Generalizations}

The construction I have described above can be generalized to include more that one, let's say $N$, DD pairs. As is well-known, boundary fermions are only available when $N$ is a power of 2 , but the general case can be described using the language of superconnections, to which the worldsheet couples through the super-Wilson line. One can also relax the requirement that the target space is topologically trivial, and include non-trivial gauge field and tachyon configurations [11]. In what follows, I will continue to assume that the target space is flat $\mathbb{C}^{r}$.

\subsection{Summary so far}

Let me now summarize this discussion $[5,6,7,11]$. B-type supersymmetry preserving boundary interactions in a Landau-Ginzburg model with polynomial bulk superpotential $W\left(x_{1}, \ldots, x_{r}\right)$ can be produced by giving a pair of $N \times N$ matrices $f\left(x_{1}, \ldots x_{r}\right), g\left(x_{1}, \ldots, x_{r}\right)$ with polynomial entries satisfying

$$
f \cdot g=g \cdot f=W \cdot \operatorname{id}_{N \times N}
$$

A solution of this equation is called a matrix factorization of $W$. The matrices $f$ and $g$ can be thought of as describing the tachyon configuration between a stack of $N$ space-filling branes and $N$ space-filling antibranes, which annihilate everywhere except at the critical points of $W$. In the supersymmetry charge, $f$ and $g$ are assembled into the odd matrix

$$
Q=\left(\begin{array}{ll}
0 & f \\
g & 0
\end{array}\right)
$$

in terms of which the requirement of $\mathcal{N}=2$ worldsheet supersymmetry can be succinctly written as the equation

$$
Q^{2}=W \cdot \operatorname{id}_{2 N \times 2 N}
$$


$Q$ acts on open strings by a supercommutator as we have seen above, and this action squares to zero by virtue of the super-Jacobi identity. Open string ground states are found by studying the cohomology classes of $Q$ acting on matrices with polynomial entries.

$$
\{Q, \Phi\}=0 \quad \Phi \equiv \Phi+\left\{Q, \Phi^{\prime}\right\}
$$

\section{Matrix Factorizations as D-brane category}

Before discussing more interesting examples, I want to explain the relevance of such constructions in string theory. The general idea is that the set of matrix factorizations provides a concrete and particularly simple example of a "D-brane category", where branes are objects, and open strings are morphisms.

The equation $Q^{2}=W$ is the condition that the boundary coupling preserve $\mathcal{N}=2$ supersymmetry. In string theory, this requirement is not sufficient for the usual applications, let's say, construction of $\mathcal{N}=1$ supersymmetric compactifications of type II theory on a Calabi-Yau with branes and fluxes, which is a starting point for many recent discussions in string phenomenology.

$\mathcal{N}=2$ worldsheet supersymmetry is sufficient, however, for one particularly important part of the story, namely (spacetime) F-terms. In fact, this follows from the general, so-called decoupling statement [27,22], which states that of the two topological string theories one can contemplate for D-branes wrapped on supersymmetric cycles, the one that can always be defined once $\mathcal{N}=2$ supersymmetry is preserved, controls the worldvolume superpotential. Here this model is the B-model. The fact that this model controls the F-terms is known for a long time (BCOV). The other model, here the A-model, which only makes sense if a certain familiar condition on the $U(1)$ R-charge is satisfied, controls the D-flatness conditions on the D-brane worldvolume.

\subsection{The grading}

Let me briefly digress on this aspect of the story. You recall that for conformal invariance in the bulk, we require the Landau-Ginzburg superpotential to be quasihomogeneous:

$$
W\left(\mathrm{e}^{i \lambda q_{i}} x_{i}\right)=\mathrm{e}^{2 i \lambda} W\left(x_{i}\right),
$$

which is equivalent to the existence of a (vector) $U(1)$ R-charge, which becomes part of the $\mathcal{N}=2$ superconformal algebra in the IR. The equivalent statement on the boundary is that there must exist an assignement of R-charge on CP spaces such that $Q$ has charge one

$$
\mathrm{e}^{i \lambda R} Q\left(\mathrm{e}^{i \lambda q_{i}} x_{i}\right) \mathrm{e}^{-i \lambda R}=\mathrm{e}^{i \lambda} Q\left(x_{i}\right)
$$

This R-charge provides an additional grading on the space of open strings,

$$
\mathrm{e}^{i \lambda R} \Phi\left(\mathrm{e}^{i \lambda q_{i}} x_{i}\right) \mathrm{e}^{-i \lambda R}=\mathrm{e}^{i \lambda q_{\Phi}} \Phi\left(x_{i}\right)
$$

where $\Phi$ is a matrix with polynomial entries, and since there is only a finite number of polynomials of fixed degree, this condition makes the problem $Q^{2}=W$ effectively finite-dimensional, in contrast to geometric versions of the same problem.

The condition that the $U(1)$ charges are integer, which is a necessary condition for the GSO projection, can be achieved as usual by orbifolding, which means giving a representation of the orbifold group $\Gamma$ on the CP space such the matrix factorization is equivariant

$$
\gamma Q\left(\gamma\left(x_{i}\right)\right) \gamma^{-1}=Q\left(x_{i}\right)
$$

(Orbifolding also produces a non-trivial K-theory of the category of matrix factorizations, which otherwise is at most torsion.)

Finally, unitarity requires that the R-charges be contained between 0 and $\hat{c}$,

$$
0 \leq q_{\Phi} \leq \hat{c}(=3)
$$

which should provide a notion of stability (at the Landau-Ginzburg point).

One can see from this that matrix factorizations are naturally equipped with all the structure for a " $D$-brane category", and this finally makes Landau-Ginzburg models a natural place to revisit the questions about such categories that have been asked many times, in the abstract setting as well as in the geometries to which the LG models are connected at large volume. (The equivalence with the large volume category has been discussed in [12].) 


\subsection{Deformations}

Here, I want to focus on the spacetime superpotential, $\mathcal{W}$, which one thinks of naively simply as the object that captures the deformation problem of $Q^{2}=W$, according to a point of view that has been taken many times in the literature, see, e.g., $[23,24,25,26]$. In fact, there are two natural questions in this context:

(i) Can we deform $Q$, holding $W$ fixed?

(ii) If we deform $W$, is there a corresponding deformation of $Q$ ?

There is a traditional answer to (i), which is that the infinitesimal deformations are given by $H^{1}$, and the obstructions by $H^{2}$ (where the grade is given by the R-charge). The usual answer to (ii) is that there is a map from infinitesimal deformations of closed strings to obstructions of open strings, and this can also lead to a superpotential.

Indeed, writing $Q=Q_{0}+\varphi \Phi$ and $W=W_{0}+\psi \Psi$, and assuming $Q_{0}^{2}=W_{0}$, one finds the equation

$$
Q^{2}-W=\varphi\{Q, \Phi\}+\varphi^{2} \Phi^{2}-\psi \Psi
$$

which expresses what we just said. The point I want to emphasize is that it can actually happen that for given bulk deformation, $\Psi$, this equation has no solution for $\Phi$, thus providing an example of a brane inducing a potential for a previously marginal bulk deformation. It should be interesting to use this kind of mechanism in the context of moduli stabilization.

\subsection{F-terms beyond perturbation theory}

Compared to other approaches, the main advantage of studying $\mathcal{W}$ via the equation $Q^{2}=W$ is that the problem of deformations of solutions, their obstructions, and even the global properties of moduli spaces of Dbranes is a finite-dimensional algebraic problem, at least up to the possibility of adding an arbitrary number of brane-antibrane pairs.

The usual approach to a problem of this sort is to use perturbation theory, with ansatz

$$
Q=Q_{0}+Q_{1}+Q_{2}+\cdots
$$

where $Q_{1}=a \in H^{1}\left(Q_{0}\right)$ and $Q_{n}$ is of order $n$ with respect to $a$. Now solving $Q^{2}=W$ recursively

$$
\begin{aligned}
& \left\{Q_{0}, Q_{2}\right\}+\left(Q_{1}\right)^{2}=0 \\
& \left\{Q_{0}, Q_{3}\right\}+\left\{Q_{1}, Q_{2}\right\}=0 \\
& \left\{Q_{0}, Q_{4}\right\}+\left\{Q_{1}, Q_{3}\right\}+\left(Q_{2}\right)^{2}=0 \\
& \vdots \\
& \left\{Q_{0}, Q_{n}\right\}+\sum_{i=1}^{n-1} Q_{i} Q_{n-i}=0
\end{aligned}
$$

requires gauge fixing at each step. Formally, matrix factorizations equip the $\mathbb{Z}$-graded vector $\operatorname{space} V^{*}=\operatorname{End}\left[x_{i}\right]$ with a differential $d=\operatorname{ad} Q_{0}$ with cohomology $H^{*}\left(Q_{0}\right)$. Gauge fixing is a choice of degree -1 -operator $u: V^{*} \rightarrow$ $V^{*-1}$ such that $P:=1-d u-u d$ is a projector on $H^{*}$.

In the unobstructed case, the formal solution is

$$
\begin{aligned}
& Q_{2}=-u\left(\left(Q_{1}\right)^{2}\right)=-u\left(a^{2}\right) \\
& Q_{3}=-u\left\{Q_{1}, Q_{2}\right\}=u\left\{a, u\left(a^{2}\right)\right\} \\
& Q_{4}=-u\left(\left\{Q_{1}, Q_{3}\right\}+\left(Q_{2}\right)^{2}\right)=-u\left\{a, u\left\{a, u\left(a^{2}\right)\right\}\right\}-u\left(u\left(a^{2}\right) \cdot u\left(a^{2}\right)\right) \\
& \text { : } \\
& Q_{n}=-u\left(\sum_{i=1}^{n-1} Q_{i} Q_{n-i}\right)=u \lambda_{n}\left(a^{\otimes n}\right)
\end{aligned}
$$


where $\lambda_{n}:\left(V^{1}\right)^{\otimes n} \rightarrow V^{2}$ is defined recursively.

Obstructions are measured by the cohomology classes

$$
\mathfrak{m}_{n}\left([a]^{\otimes n}\right):=\left[\lambda_{n}\left(a^{\otimes n}\right)\right] \in H^{2}\left(M_{0}, M_{0}\right) .
$$

( $\mathfrak{m}_{n}$ are the higher products of an $A_{\infty}$ structure.)

In the general (obstructed) case,

$$
Q=Q_{0}+a+\sum_{n=2}^{\infty} u \lambda_{n}\left(a^{\otimes n}\right)
$$

satisfies

$$
Q^{2}=W_{0}-\sum_{n=2}^{\infty} \mathfrak{m}_{n}\left(a^{\otimes n}\right)-\sum_{\ell=1}^{\infty}(-1)^{\ell} u \underbrace{[\Delta Q \ldots u[\Delta Q}_{\ell}, \sum_{n=2}^{\infty} \mathfrak{m}_{n}\left(a^{\otimes n}\right)] \ldots],
$$

where $\Delta Q=Q-Q_{0}$. The F-term equations are (assuming, for simplicity, that $W=W_{0}$ is fixed)

$$
\sum_{n=2}^{\infty} \mathfrak{m}_{n}\left(a^{\otimes n}\right)=0 \quad \in H^{2}\left(Q_{0}\right)
$$

In principle, up to $\operatorname{dim}\left(V^{1}\right)<\infty$ terms can appear. In practice, perturbation theory closes on a small number of operators, $A_{1}, \ldots, A_{N}$. One can then study the complete problem without resorting to a perturbative expansion (and without the need for gauge fixing). Indeed, the ansatz $\left(n=\operatorname{dim} H^{1}\right)$

$$
\Delta Q=Q-Q_{0}=\sum_{i=1}^{n} \phi_{i} \Phi_{i}+\sum_{I=1}^{N} a_{I} A_{I}
$$

satisfies

$$
Q^{2}-W_{0}=P(\Delta Q)^{2}+d\left(\Delta Q+u(\Delta Q)^{2}\right)+u d Q^{2}
$$

(where we used $P=1-u d+d u$ ). We obtain three equations

$$
\begin{aligned}
P(\Delta Q)^{2} & =0 \\
d\left\{\Delta Q+u(\Delta Q)^{2}\right\} & =0 \\
u d\left(Q^{2}\right) & =0 .
\end{aligned}
$$

Perturbation theory amounts to neglecting $u d\left(Q^{2}\right)=0$, which is self-consistent to all orders in the perturbation. In general, however, one might obtain new solutions which are invisible in perturbation theory. Matrix factorizations are a framework in which the more complete treatment of such problems is possible.

\section{Example 1 - The torus}

A Landau-Ginzburg model for the two-dimensional torus can be built on the LG potential

$$
\left(W=x^{3}+y^{3}+z^{3}+\psi x y z\right) / \mathbb{Z}_{3}
$$

where $\psi$ is the complex structure parameter of the torus. Consider the matrix

$$
A=\left(\begin{array}{ccc}
\alpha x & \beta z & \gamma y \\
\gamma z & \alpha y & \beta x \\
\beta y & \gamma x & \alpha z
\end{array}\right)
$$

We see that

$$
\operatorname{det} A=\left(\alpha^{3}+\beta^{3}+\gamma^{3}\right) x y z-\alpha \beta \gamma\left(x^{3}+y^{3}+z^{3}\right)
$$

which is equal to $\lambda W$ with

$$
\lambda=-\alpha \beta \gamma
$$

if and only if

$$
\alpha^{3}+\beta^{3}+\gamma^{3}+\psi \alpha \beta \gamma=0 .
$$


Thus, if we let $B$ be the adjoint of $A$ (the matrix of subdeterminants) up to a factor,

$$
\begin{aligned}
B & :=\frac{1}{\lambda} \operatorname{adj}(A) \\
& =-\frac{1}{\alpha \beta \gamma}\left(\begin{array}{ccc}
\alpha^{2} y z-\beta \gamma x^{2} & \gamma^{2} x y-\alpha \beta z^{2} & \beta^{2} x z-\alpha \gamma y^{2} \\
\beta^{2} x y-\alpha \gamma z^{2} & \alpha^{2} y z-\beta \gamma y^{2} & \gamma^{2} y z-\alpha \beta x^{2} \\
\gamma^{2} x z-\alpha \beta y^{2} & \beta^{2} y z-\alpha \gamma x^{2} & \alpha^{2} x y-\beta \gamma z^{2}
\end{array}\right)
\end{aligned}
$$

Then we find

$$
A B=B A=W \mathrm{id},
$$

as long as $(\alpha, \beta, \gamma)$ obeys (51) and $\alpha \beta \gamma$ is non-zero. The moduli space of this brane on the torus is thus isomorphic to the torus itself, as expected of any B-type D-brane on the torus.

What happens as $\lambda \rightarrow 0$, where the matrix factorization becomes naively singular? The trick here is to add a trivial brane-antibrane pair

$$
f=\left(\begin{array}{cc}
-\frac{1}{\alpha} W & 0 \\
0 & A
\end{array}\right) \quad g=\left(\begin{array}{cc}
-\alpha & 0 \\
0 & B
\end{array}\right)
$$

and make a gauge transformation on $\mathrm{CP}$ spaces that removes the singular part of $B$.

\section{Example 2 - The quintic}

The "mirror quintic" Landau-Ginzburg model is

$$
\left(W=x_{1}^{5}+x_{2}^{5}+x_{3}^{5}+x_{4}^{5}+x_{5}^{5}+\psi x_{1} x_{2} x_{3} x_{4} x_{5}\right) /\left(\mathbb{Z}_{5}\right)^{4}
$$

At $\psi=0$, an interesting factorization of this $W$ can be obtained by taking the tensor product of minimal model factorizations I discussed before

$$
Q_{0}=\left(\begin{array}{cc}
0 & x_{1}^{2} \\
x_{1}^{3} & 0
\end{array}\right) \oplus\left(\begin{array}{cc}
0 & x_{2}^{2} \\
x_{2}^{3} & 0
\end{array}\right) \oplus\left(\begin{array}{cc}
0 & x_{3}^{2} \\
x_{3}^{3} & 0
\end{array}\right) \oplus\left(\begin{array}{cc}
0 & x_{4}^{2} \\
x_{4}^{3} & 0
\end{array}\right) \oplus\left(\begin{array}{cc}
0 & x_{5}^{2} \\
x_{5}^{3} & 0
\end{array}\right)
$$

It turns out that this has exactly one marginal operator

$$
\Phi=\left(\begin{array}{cc}
0 & 1 \\
-x_{1} & 0
\end{array}\right) \otimes\left(\begin{array}{cc}
0 & 1 \\
-x_{2} & 0
\end{array}\right) \otimes\left(\begin{array}{cc}
0 & 1 \\
-x_{3} & 0
\end{array}\right) \otimes\left(\begin{array}{cc}
0 & 1 \\
-x_{4} & 0
\end{array}\right) \otimes\left(\begin{array}{cc}
0 & 1 \\
-x_{5} & 0
\end{array}\right)
$$

as well as one obstruction

$$
\Psi=x_{1} x_{2} x_{3} x_{4} x_{5} \cdot \mathrm{id}
$$

which is exactly the marginal bulk deformation. One can also easily see that

$$
\Phi^{2}=-\Psi
$$

So, $Q=Q_{0}+\varphi \Phi$ will square to $W=W_{0}+\psi \Psi$ iff

$$
\varphi^{2}+\psi=0
$$

This is the F-flatness equation on the D-brane worldvolume, which one may integrate to the superpotential

$$
\mathcal{W}=\frac{1}{3} \varphi^{3}+\varphi \psi
$$

(This confirms a prediction of $[27,28]$.) Treating $\psi$ as a closed string parameter, we learn that except at $\psi=0$, our brane has two supersymmetric vacua. The coalescence of the two vacua at $\psi=0$ is accompagnied by the appearance of an additional massless open string field $\Phi$. 


\subsection{A mirror symmetry interpretation}

In conclusion, I want to offer a geometric interpretation involving the mirror geometry, which here is the Fermat quintic in $\mathbb{C P}^{4}$

$$
X=\left\{z_{1}^{5}+z_{2}^{5}+z_{3}^{5}+z_{4}^{5}+z_{5}^{5}=0\right\} \subset \mathbb{C P}^{4}
$$

Closed string mirror symmetry gives the map between the marginal closed string operator $\Psi$ and the generator of $H^{2}(X, \mathbb{Z})$, or in other words, between the complex structure parameter $\psi$ and the Kähler parameter $t$ of $X$. The Yukawa coupling which on the B-model side can be computed to be

$$
\kappa_{\psi \psi \psi}=\frac{1}{5^{5}+\psi^{5}}
$$

can then be expanded on the A-model side

$$
\kappa_{t t t}=5+\sum(\text { worldsheet instantons) }
$$

where the 5 is the result from classical geometry, and the sum over holomorphic spheres in $X$ is a power series in $q=\exp (-t)$.

What about open string mirror symmetry? One can show $[27,29]$ that the brane I have been discussing in the Landau-Ginzburg model is mirror to a familiar special Lagrangian cycle in $X$, namely the real locus of $X$,

$$
L=\left\{z \in X, z_{i} \in \mathbb{R} \forall i\right\}
$$

$L$ is topologically an $\mathbb{R} P^{3}$. Since $H_{1}\left(\mathbb{R} \mathbb{P}^{3}\right)=\mathbb{Z}_{2}$, a brane wrapped on $\mathbb{R} \mathbb{P}^{3}$ has two vacua, distinguished by a discrete Wilson line. These can be identified with the two vacua we had found for the Landau-Ginzburg brane. To identify the field that becomes massless at $\psi=0$, we have to recall that the zero modes of strings wrapped on special Lagrangians is determined not by ordinary cohomology but by Floer cohomology, which differs from ordinary cohomology by a sum over holomorphic discs ending on $L$. In our context, the relevant complex is

$$
C^{0} \stackrel{0}{\longrightarrow} C^{1} \stackrel{\delta}{\longrightarrow} C^{2} \stackrel{0}{\longrightarrow} C^{3}
$$

where for ordinary cohomology, all $C^{i} \cong \mathbb{Z}$, and $\delta=2$. For Floer cohomology, we will have (after tensoring the complex with the appropriate coefficient field)

$$
\delta=2+\sum(\text { holomorphic discs) }
$$

where the sum over holomorphic discs should be a power series in $q^{1 / 2}=\exp (-t / 2)$, because the discs have half the volume of the spheres. At large volume $t \rightarrow \infty$, the cohomology is trivial except in degree 0 and 3 . But if $\delta=0$ for some value of $t$ (after analytic continuation), there will be an additional massless field in degree 1 . The conjecture is that this is precisely what happens at $\psi=0$, the additional massless field being $\Phi$.

Of course, making this proposal more precise depends on identifying the correct map between the (generally) massive field $\Phi$ on the Landau-Ginzburg side and the integral generator of $C^{1}$ in the Floer complex.

\section{Summary and Outlook}

We have seen that matrix factorizations are a useful B-type model for topological D-branes on Calabi-Yau manifolds. In particular, they allow simple calculations of worldvolume superpotentials for D-branes wrapped on supersymmetric cycles. When combined with orientifolds (see, e.g., [29]), this will allow a rather more systematic investigation of some properties of $\mathcal{N}=1$ string vacua. We have also seen that the approach holds some promises toward realizing open string mirror symmetry for compact Calabi-Yau manifolds (see [30] for some recent progress in this direction).

The computation of the superpotential also illustrates that one may view $Q^{2}=W$ as a finite-dimensional model of (background-independent, topological) string field theory, which would be interesting to explore further.

One aspect of the story that we have not mentioned here is the direct connection to geometry in the B-model. There exist mathematical constructions that relate matrix factorizations of $W$ to bundles on the hypersurface $\{W=0\}$ in the corresponding weighted projective space. It would be interesting to realize such connections via physical models of the linear sigma model type. 
Acknowledgements J.W. would like to thank C. Bachas, E. Cremmer, and P. Windey for the invitation to speak at Strings '04, a most wonderfully organized conference. The work of K.H. was supported by Alfred P. Sloan Foundation, Connaught Foundation and NSERC. The research of J.W. was supported in part by the National Science Foundation under Grant No. PHY99-07949.

\section{References}

[1] E. J. Martinec, "Algebraic Geometry And Effective Lagrangians," Phys. Lett. B 217 (1989) 431.

[2] C. Vafa and N. P. Warner, "Catastrophes And The Classification Of Conformal Theories," Phys. Lett. B 218, 51 (1989).

[3] C. Vafa, "String Vacua And Orbifoldized L-G Models," Mod. Phys. Lett. A 4 (1989) 1169.

[4] C. Vafa, "Topological Landau-Ginzburg Models," Mod. Phys. Lett. A 6 (1991) 337.

[5] A. Kapustin and Y. Li, "D-branes in Landau-Ginzburg models and algebraic geometry," JHEP 0312, 005 (2003) arXiv:hep-th/0210296 .

[6] I. Brunner, M. Herbst, W. Lerche and B. Scheuner, "Landau-Ginzburg realization of open string TFT," arXiv:hep-th/0305133

[7] A. Kapustin and Y. Li, "Topological correlators in Landau-Ginzburg models with boundaries," Adv. Theor. Math. Phys. 7, 727 (2004) arXiv:hep-th/0305136.

[8] D. Eisenbud, "Homological algebra on a complete intersection, with an application to group representations," Trans. Amer. Math. Soc. 260 (1980)

[9] K. Hori and J. Walcher, "F-term equations near Gepner points," arXiv:hep-th/0404196

[10] A. Kapustin and Y. Li, "D-branes in topological minimal models: The Landau-Ginzburg approach," JHEP 0407 (2004) 045 arXiv:hep-th/0306001.

[11] C. I. Lazaroiu, "On the boundary coupling of topological Landau-Ginzburg models," arXiv:hep-th/0312286

[12] S. K. Ashok, E. Dell'Aquila and D. E. Diaconescu, "Fractional branes in Landau-Ginzburg orbifolds," arXiv:hep-th/0401135

[13] K. Hori, "Boundary RG flows of $N=2$ minimal models," arXiv:hep-th/0401139

[14] S. K. Ashok, E. Dell'Aquila, D. E. Diaconescu and B. Florea, "Obstructed D-branes in Landau-Ginzburg orbifolds," arXiv:hep-th/0404167

[15] M. Herbst and C. I. Lazaroiu, "Localization and traces in open-closed topological Landau-Ginzburg models," arXiv:hep-th/0404184

[16] M. Herbst, C. I. Lazaroiu and W. Lerche, "D-brane effective action and tachyon condensation in topological minimal models," arXiv:hep-th/0405138

[17] N. P. Warner, "Supersymmetry in boundary integrable models," Nucl. Phys. B 450, 663 (1995) hep-th/9506064.

[18] S. Govindarajan, T. Jayaraman and T. Sarkar, "Worldsheet approaches to D-branes on supersymmetric cycles," Nucl. Phys. B 580 (2000) 519 hep-th/9907131.

[19] K. Hori, A. Iqbal and C. Vafa, "D-branes and mirror symmetry," hep-th/0005247

[20] K. Hori, "Linear models of supersymmetric D-branes," hep-th/0012179

[21] S. Hellerman, S. Kachru, A. E. Lawrence and J. McGreevy, "Linear sigma models for open strings," JHEP 0207, 002 (2002) hep-th/0109069.

[22] M. R. Douglas, "D-branes, categories and N = 1 supersymmetry," J. Math. Phys. 42, 2818 (2001) arXiv:hep-th/0011017.

[23] C. I. Lazaroiu, "String field theory and brane superpotentials," JHEP 0110 (2001) 018 arXiv:hep-th/0107162.

[24] A. Tomasiello, "A-infinity structure and superpotentials," JHEP 0109, 030 (2001) arXiv:hep-th/0107195.

[25] M. R. Douglas, S. Govindarajan, T. Jayaraman and A. Tomasiello, "D-branes on Calabi-Yau manifolds and superpotentials," arXiv:hep-th/0203173

[26] S. Kachru, S. Katz, A. E. Lawrence and J. McGreevy, "Open string instantons and superpotentials," Phys. Rev. D 62, 026001 (2000) arXiv:hep-th/9912151.

[27] I. Brunner, M. R. Douglas, A. E. Lawrence and C. Romelsberger, "D-branes on the quintic," JHEP 0008, 015 (2000) arXiv:hep-th/9906200.

[28] I. Brunner and V. Schomerus, "On superpotentials for D-branes in Gepner models," JHEP 0010 (2000) 016 arXiv:hep-th/0008194. 
[29] I. Brunner, K. Hori, K. Hosomichi and J. Walcher, "Orientifolds of Gepner models," arXiv:hep-th/0401137

[30] I. Brunner, M. Herbst, W. Lerche and J. Walcher, "Matrix factorizations and mirror symmetry: The cubic curve," arXiv:hep-th/0408243 